\title{
Téoros
}

Revue de recherche en tourisme

\section{Salvador de Bahia}

Réflexions sur une cité mystique à résoudre

\section{Yvan Desbiens}

Volume 23, numéro 3, automne 2004

URI : https://id.erudit.org/iderudit/1071234ar

DOI : https://doi.org/10.7202/1071234ar

Aller au sommaire du numéro

Éditeur(s)

Université du Québec à Montréal

ISSN

0712-8657 (imprimé)

1923-2705 (numérique)

Découvrir la revue

\section{Citer cet article}

Desbiens, Y. (2004). Salvador de Bahia : réflexions sur une cité mystique à résoudre. Téoros, 23(3), 55-61. https://doi.org/10.7202/1071234ar d'utilisation que vous pouvez consulter en ligne.

https://apropos.erudit.org/fr/usagers/politique-dutilisation/ 


\section{Salvador de Bahia \\ Réflexions sur une cité mystique à résoudre}

\section{Yvan Desbiens}

En fonction de l'importance prédominante du Brésil en Amérique latine, comme pays en voie de développement (Mauro, 1975 : 77-87), et de son potentiel de ressources naturelles riches et diversifiées, l'analyse de la ville de Salvador de Bahia au nord-est du Brésil exige une mise en relation incontournable des dimensions historiques, coloniales et sociologiques dans la compréhension du pluralisme de la réalité brésilienne. Cet article dépeint quelques caractéristiques urbaines de la ville de Salvador et questionne sa particularité sociale contradictoire au-delà des limites physiologiques et des facteurs ambiants de la cité. La question se pose à savoir si l'identité culturelle de Salvador peut souffrir le progrès industriel et garder son cachet historique et folklorique. On peut croire que l'enrichissement collectif nécessaire de sa population passe par une diversification des activités et une consolidation de son influence culturelle et économique au Brésil. Cette émancipation économique se produira par une promotion des intentions et des valeurs qui animent les citoyens de la ville. L'historicité riche et unique de la ville a incité l'UNESCO à reconnaître la ville « patrimoine culturel mondial », décision qui consacre bien la valeur sociologique et la particularité exceptionnelle du site urbain. Cette accréditation donne l'occasion à la Préfecture ou la Mairie de miser sur cette reconnaissance internationale. $\mathrm{La}$ participation originale et culturelle de la population noire (héritée de l'époque coloniale esclavagiste) accentue le fait qu'on puisse encore la surnommer la Perle Noire du Brésil ${ }^{1}$.

\section{Salvador : espace et société}

Au centre du Reconcavo Bahiano, qui ceinture la baie de Todos Santos, l'agglomération est une porte maritime de distribution de sucre et de produits agricoles de cet amphithéâtre formé par les basses terres littorales atlantiques et le plateau semi-aride du Sertão.

Sa population, produit d'une convergence ethnique et culturelle (métissage généralisé), profite d'un milieu naturel agréable; elle bénéficie vraisemblablement de la grâce de tous les saints. Cet alliage racial et culturel (historiquement encouragé par les colonisateurs portugais) a produit des assises solides à l'unité nationale (unilinguisme portugais un vif sentiment patriotique).

Le tempérament de la majorité bahianaise se fonde sur des principes existentiels, où les problèmes se résolvent souvent par accommodation culturelle (la religion, la musique, la poésie, le partage). L'esprit cartésien et le capitalisme dur y voisinent avec la relativité du temps, le mercantilisme artisanal et l'improvisation. La sérénité des habitants, la douceur climatique, l'omniprésence des plages et des cocotiers accentuent le caractère touristique de Salvador. La métropole et capitale de Bahia, avec ses 2,5 millions d'habitants, baigne dans un système climatique qui conjugue le soleil tropical et la fraîcheur maritime (moyenne annuelle de 25 degrés). Ses quartiers se tassent le long des interminables plages sablonneuses de l'Atlantique.

Milton Santos (1968: 58) définit Salvador comme une métropole incomplète, sans toutefois préciser la nature spécifique d'une métropole véritable ; malgré tout, avec une telle

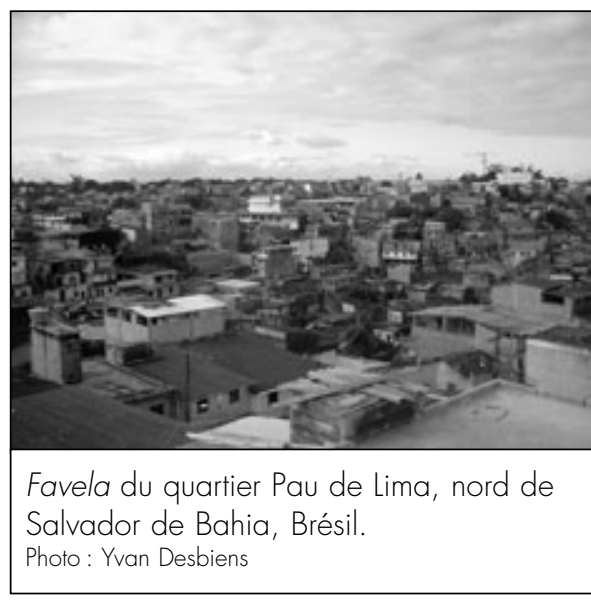

progression dans toutes les sphères d'activité (industrielle, commerciale, administrative), Salvador se perçoit sans contredit comme la véritable métropole du Nordeste. Première capitale du Brésil de 1549 à 1763, elle a perdu ce titre au profit de Rio de Janeiro, l'administration publique et l'activité économique se déplaçant vers le Sudeste (Rio de Janeiro, São Paulo, Belo Horizonte). À partir du dix-neuvième siècle, comparativement à l'enrichissement et à l'industrialisation du Sudeste, le Nordeste, axé surtout sur l'agriculture, perdait d'autant son prestige politique et économique. Comme en Amérique du Nord (États-Unis, Canada), l'immigration européenne (Allemands, Italiens, Portugais, etc.) a joué un rôle dans l'occupation et l'expansion du territoire brésilien. Cette immigration blanche a donné naissance à un réseau urbain et industriel majeur profitable au Sudeste. La décision gouvernementale de construire l'ambitieuse capitale de Brasilia, inaugurée en 1960 par le président Kubitschek, consacrait le fait que l'administration publique se situerait dorénavant à l'extérieur du rayonnement de la ville de Salvador de Bahia et du Nordeste. 
FIGURE 1: Accroissement de population des villes de Salvador, Belo Horizonte, Rio de Janeiro, Sao Pãulo

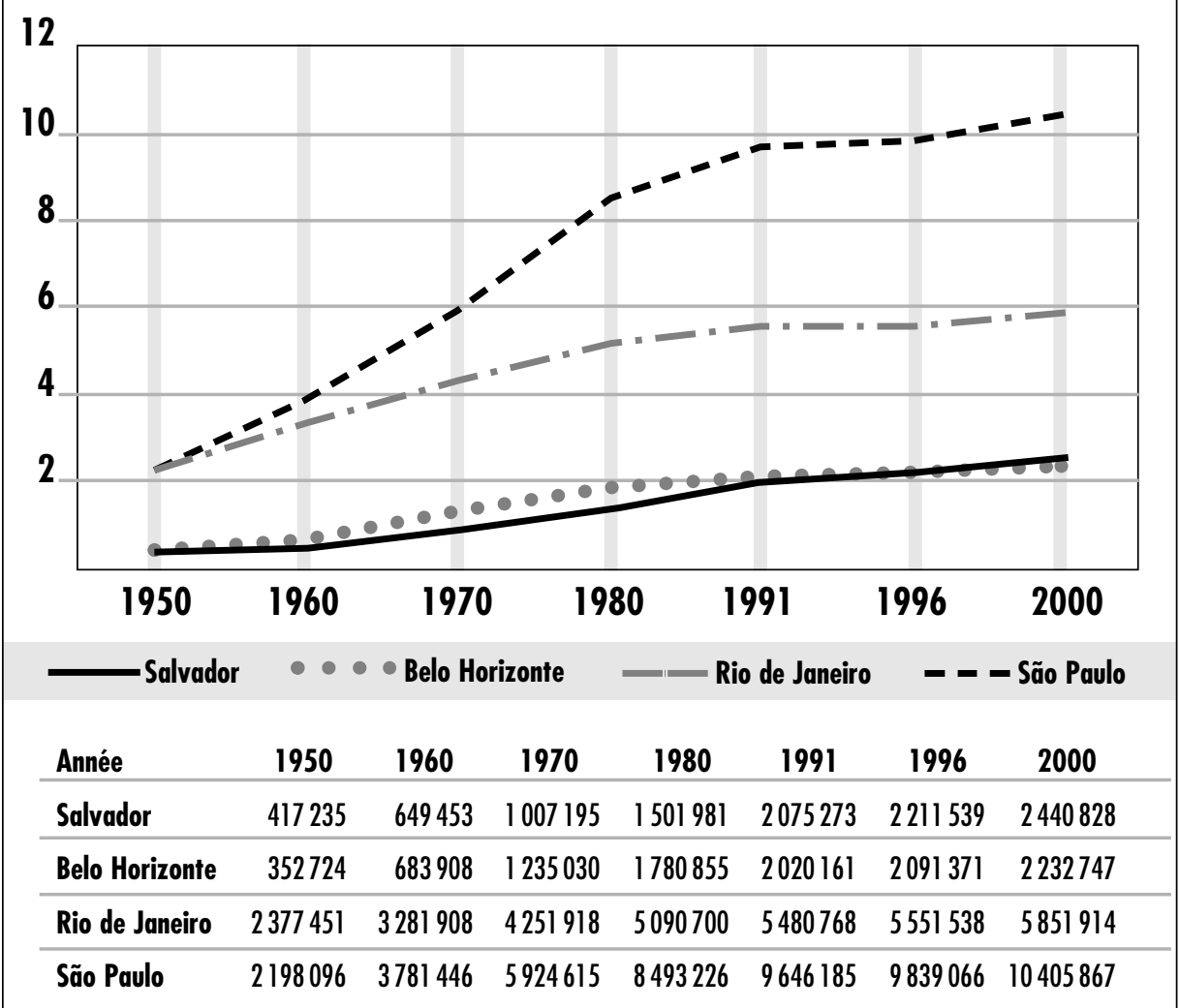

Source : IBGE

\section{FIGURE 2: Accroissement de population de Salvador entre 1950-2000}

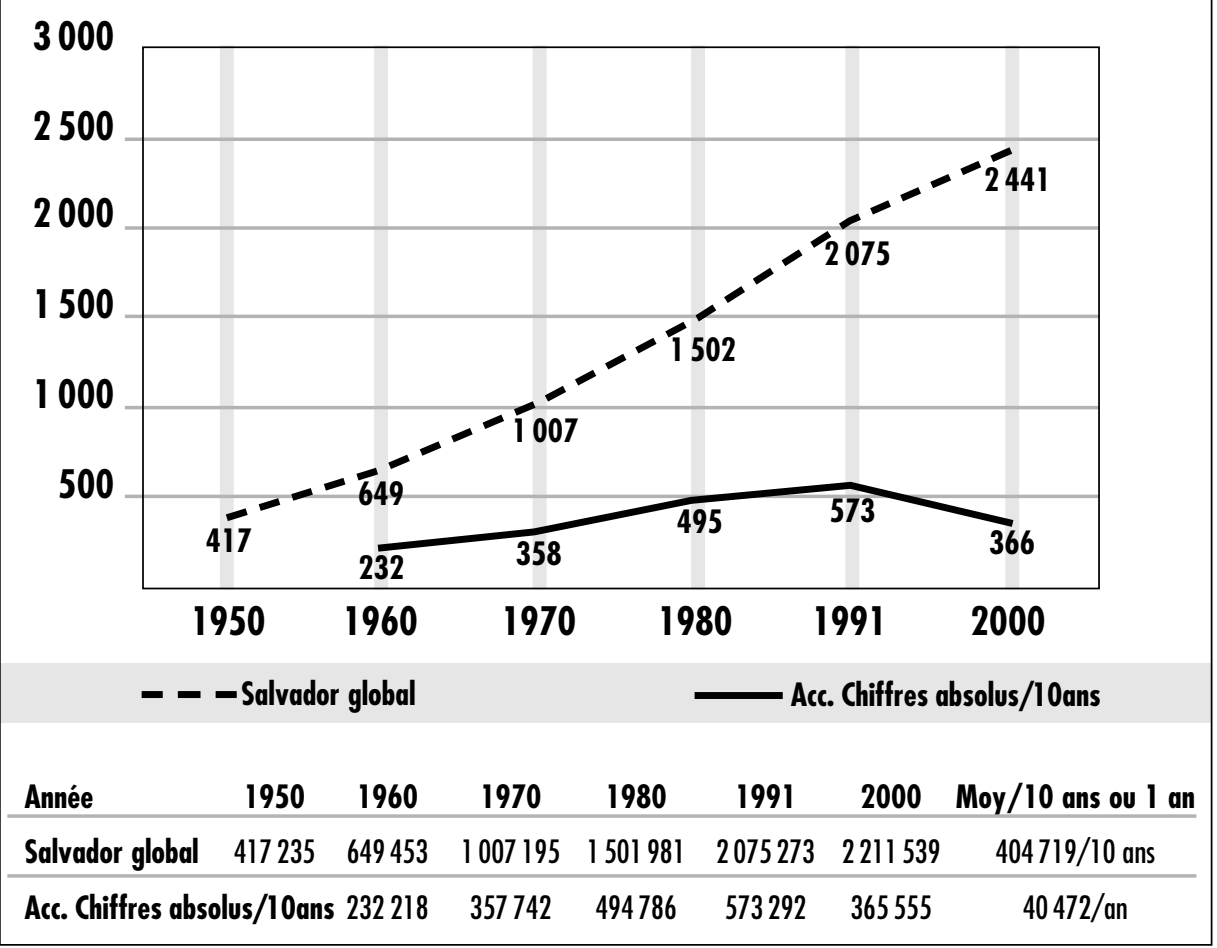

Source : IBGE
Le coefficient de corrélation des taux d'accroissement de population entre les principales agglomérations brésiliennes (Salvador, Belo Horizonte, Rio de Janeiro, São Paulo) indique pendant la période 1950-2000 un ratio d'accroissement similaire, si ce n'est de l'envol de la population de São Paulo, en chiffres absolus de deux à plus de dix millions. La courbe démographique de Salvador recoupe et dépasse à peine celle de Belo Horizonte depuis les années 1980, mais cette progression désigne un recoupement démographique étonnant qui illustre certainement le dynamisme de la ville malgré la force économique de la région du Brésil Sud (Brasul), en opposition au Brésil Nord (Brasnorte).

Comparativement aux trois autres villes, qui voient leur population se multiplier par six, Rio de Janeiro triple sa population. Les quatre villes connaissent une accélération de leur population à partir des années 1960, puis un ralentissement à partir des années 1990.

Le ralentissement semble être davantage significatif pour Rio de Janeiro. En moyenne, la population de Salvador s'accrôit de 40000 habitants par année, donc un taux moyen d'accroissement des résidants de $3,5 \%$ par année, taux supérieur au taux d'accroissement du pays $(2,5 \%)$. La période précédente de 1960-1990 correspond à un rattrapage de la zone d'influence de Salvador, par l'implantation à la périphérie nord d'industries primaires et secondaires, avec la collaboration de l'État. L'économie d'échelle aurait permis ensuite une croissance dans le secteur commercial, celui de la construction et des services. La baisse du taux de croissance démographique, à partir des années 1990, correspond à une période de morosité économique. Durant cette période, la ville devrait sûrement être moins captivante, en temps de stagnation économique nationale et internationale (dévaluation de la monnaie, inflation, chômage etc.) ; pourtant, elle continue quand même à connaître un taux d'accroissement dépassant la moyenne nationale de $1,0 \%$ par année. L'agglomération fait partie de ce phénomène mondial d'urbanisation. Elle demeure à la fois progressive (du moins démographiquement) et attrac- 
tive dans sa vocation de ville touristique et d'offre de services. La ville simultanément profite des avantages d'une consommation et d'une production accrues, mais endure aussi les obligations de fournir une infrastructure urbaine suffisante.

En fonction du taux accéléré d'accroissement démographique, l'absorption de l'excédent démographique (naturelle et migratoire) amplifie l'urgence des investissements sociaux, qui étaient déjà en deçà des besoins. Durant la période de latence et de réorganisation, la ville, victime de son propre dynamisme qui attire en majorité les ruraux les plus pauvres et les plus démunis, supporte une pression intolérable afin de répondre aux besoins sociaux accrus (logements, travail, santé, éducation). Le Nordeste a connu, depuis 1970 jusqu'à récemment, une sécheresse persistante qui repoussait les ruraux vers la ville. C'est pourquoi Santos (1968: 57) mentionne que les villes de Salvador, de Recife, de Belém et même de Rio ont une faculté d'absorber, par une masse énorme de sous-emplois ou non-emplois déguisés, une foule d'illettrés.

La ville de Salvador est une ville dortoir récréative, culturelle, administrative, touristique et son nouveau poids démographique en fait un centre commercial métropolitain tourné autant vers le marché interne qu'externe. Elle devient la métropole du Nordeste, selon les indices récents de sa progression économique et démographique. Son dynamisme est surtout fondé sur de riches événements et des circonstances historiques dans un lieu physiquement agréable. Le promontoire péninsulaire, où se situe la ville, s'avance comme un éperon dans la mer et laisse à penser, en considération des nombreux vestiges et des forts disséminés sur le littoral, que le site répondait bien à une stratégie militaire. Les parties les plus anciennes occupent l'extrémité sud-ouest de la péninsule (Barra, le centre historique ou Centro Mercado, Centro Modelo, Pelourinho) dominant la Baie de Todos os Santos (Tous les Saints). Ce secteur révèle l'empreinte des conquérants (portugais surtout, français et hollandais) par l'architecture baroque et byzantine.
Si son site promontoire et péninsulaire tropical a favorisé et suscité le développement de Salvador de Bahia, les conditions sociales méritent d'y être analysées et interprétées lorsqu'elles s'inscrivent régulièrement dans ses murs, ses rues, ses quartiers. Cet enchaînement récursif et généralisé du profil urbain de la ville dessine un plan périodique, affirmant le dualisme du développement urbain moderne, avec un entassement populaire improvisé qui crée des tensions entre la richesse et la pauvreté. Cette discontinuité sociale, de l'organisé à l'improvisé, exprime un besoin spontané et urgent chez les nouveaux arrivants d'occuper un espace vital (favelas ou bidonvilles) près des centres d'activités commerciales ou industrielles. Par l'étendue de ce type d'occupation, le phénomène d'occupation démographique asymétrique est ancien ; il se perpétue malgré qu'il soit un comportement social politiquement difficile à contrôler. Ce type d'occupation n'est pas particulier à l'État de Bahia, mais en extension spatiale nationale et intégrée au processus historique d'urbanisation/industrialisation brésilien (Dos Passos, 1964 : 41). Un tel type d'occupation territoriale, qu'il se généralise sur l'espace brésilien ou ailleurs dans le monde, crée des quartiers de démunis, des bidonvilles transitoires. Cependant, ce mode d'habitat, au Brésil, se particularise par son dynamisme naturel désarmant, parce qu'il est évolutif et démocratisé. Il résulte de la mouvance et du mode de vie des populations autorisés par l'immensité territoriale et par l'abondance des ressources du Brésil. Les conditions physiques, la colonisation, la conquête d'un territoire immense ont exigé un nomadisme et une intégration raciale et culturelle typiques.

Dans un sens, le déplacement des populations à l'image des chercheurs de métaux précieux et d'esclaves (bandeiras) et les agriculteurs sur brûlis ont introduit ce goût de la mobilité. Dans un autre sens, les propriétaires de grands domaines

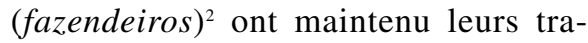
vailleurs agricoles dans la pauvreté et l'analphabétisme ${ }^{3}$, sans égard au potentiel humain comme facteur de productivité nationale. Cette concentration de la terre et le servage traditionnel des travailleurs

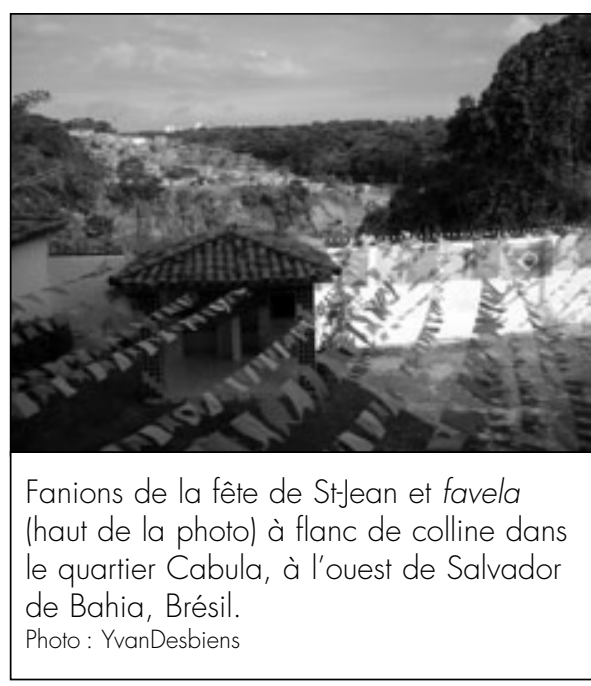

ruraux tels les cueilleurs saisonniers (boias frias) (Martins, 1998 : 147) ont produit un capital humain particulier perpétué dans les grands domaines agricoles (latifundios) (Smith, 1972: 351-355).

De cause à effet, repoussés des campagnes, les migrants de l'arrière-pays transportent, avec leur misère et leur ignorance, des modes de survie traditionnels incompatibles avec une adaptation heureuse à un espace urbain aussi restreint. Certains amérindiens de l'intérieur, comme modèle de cas, bâtissent des habitations sur pilotis en bordure des cours d'eau. Évidemment, cette habitude n'a pas la même inci dence en forêt, avec un éparpillement des populations. Sur le littoral et en bordure des villes, où le ramassage des ordures et le traitement de l'eau est déjà un problème, cette pratique, en plus de provoquer des effets écologiques désastreux, perturbe l'activité récréative balnéaire (quartiers de Ribeira et d'Itapagipe face à la baie Dos Tainheiros) et repousse le touriste, affaiblissant un secteur économique moteur et une source de revenus. Dans une ville déjà surpeuplée, la concurrence et les problèmes sociaux (hygiène, santé, violence) augmentent proportionnellement à la densité. En raison de l'attitude fataliste et tolérante de la ville (les policiers ne pénètrent habituellement pas dans les favelas, les touristes non plus, à moins d'être accompagnés par des familiers), les favelas s'améliorent lentement au gré des interventions ponctuelles étatiques en éducation, en 
santé, en logement, etc. Pour s'adapter au phénomène, les propriétaires se voient peu à peu pressés de se protéger contre la violence compréhensible, imminente, prévisible du démuni. La faim et l'indigence font parfois surgir des cobras $d u$ panier, le marché du travail souffre à procurer de l'emploi à tous.

Lors des nombreuses fêtes religieuses et des carnavals, par une récupération de rites africains, les saints de la chrétienté se trouvent subtilement incorporés aux divinités

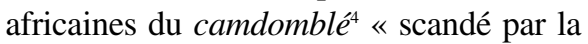
axé unsic $^{5} \gg$ et aux rituels vaudous (Agier, 1999 : 298). Ce mysticisme teinte l'humeur populaire d'une insouciance qui laisse mal deviner une problématique sociale sous-jacente, cette vertu mystique de l'être cache une possible volonté d'identification et d'affirmation ethniques (Walniez et Brustlein, 2000 : 111). Le futebol (soccer), la fête, la religion décorent un quotidien marqué davantage par le salaire minimum (un peu plus de 100 réaux/mois); les frais de transport et les autres dépenses incontrôlables et inabordables rendent indispensables l'usage de la favela et de la plage.

\section{Urbanisation et transformations sociales à Salvador}

La préfecture est le principal intervenant dans le développement socio-économique de Salvador. Le gouvernement fédéral et l'État interviennent, mais c'est la mairie qui demeure responsable des programmes d'action sociale. La préfecture doit s'appuyer sur ses avantages balnéaires et historiques (églises, forteresses, bâtiments anciens).

Malgré l'industrie naissante, la pétrochimie dans la périphérie, à Camacari, trente kilomètres au nord, Salvador se manifeste surtout comme une ville d'activités tertiaires (commerce, administration, tourisme $)^{6}$. Le paysage et le chatoiement de son folklore traditionnel et de ses nombreuses fêtes alimentent en grande partie l'attrait et la richesse municipale.

Il ressort qu'un meilleur étalement de la richesse collective s'assujettit à une gestion orientée vers l'exploitation de la ressource environnementale et de la ressource culturelle. La protection civile, le maintien de la salubrité et de la sécurité des plages, la rénovation et la conservation des sites historiques, le développement des arts et de l'artisanat, la croissance et la diversification de l'industrie manufacturière, la promotion de la santé et l'éducation solliciteront éventuellement l'innovation et la créativité de la classe dirigeante et des investissements de la préfecture de Salvador. Des usines de montage et de haute technologie pourraient fort bien s'y installer, considérant la maind'œuvre disponible et les nombreuses maisons de formation, mais cela ne semble pas se produire et le secteur manufacturier stagne et s'essouffle comparativement au secteur commercial et à celui de la construction.

Salvador peine à fournir un transport en commun adéquat. Le parachèvement du métro (gestion pénible et douteuse) enlèverait la congestion aux heures de pointe; le réseau d'autobus est, somme toute, acceptable et polyvalent. À vrai dire, le transport en commun reste dispendieux pour les travailleurs et les étudiants. C'est particulièrement ces derniers qui réussissent joyeusement, par de nombreux embouteillages (engarrafamento) et manifestations, à réduire la hausse des prix. Les mouvements syndicaux sont singulièrement passifs ou absents, la nature précaire des emplois (commerce de rue, secteur tertiaire) ne se prêtant pas à la syndicalisation de masse.
La ville a hérité à la fois des bienfaits et des malheurs du colonialisme, des œuvres audacieuses et magnifiques, mais aussi de l'afflux de population rurale (noire, métis, autochtones...) nombreuse et pauvre de l'arrière-pays (ressacs de la région rurale du São Francisco). L'armée paysanne du mouvement des sans-toit ou sans-terre (sem-teto ou sem-terra) (Soares,de Souza 1999: 120-127) revendique le partage des terres contre les grands propriétaires (latifundiaires) depuis 1981. Leurs minuscules cabanes de bambou s'alignent le long des principales routes de Bahia, illustrant bien que la plupart des migrants sont dépourvus de tout, comme la plupart de ceux qui s'installent en ville. Cette rétroaction perverse, continental-ville, contrebalance les effets de l'industrialisation, de la détérioration des écosystèmes intérieurs par la monoculture et l'exploitation abusive de la ressource (déforestation, épuisement des sols...). En plus d'un taux de natalité élevé, le défi immense d'intégrer ces nouveaux venus sans heurts ni difficulté relève de prouesses humanitaires sans précédent des villes, des États, des entreprises, des organismes mondiaux. L'urbanisation alimentée par la campagne, comme dans le reste de la planète, cause l'hypertrophie chronique des villes et des pressions dans la demande de revenus, de besoins essentiels comme les transports, les services de santé, l'éducation, etc. Souvent, les plus faibles, dont les enfants et les femmes, sont socialement les premières victimes.

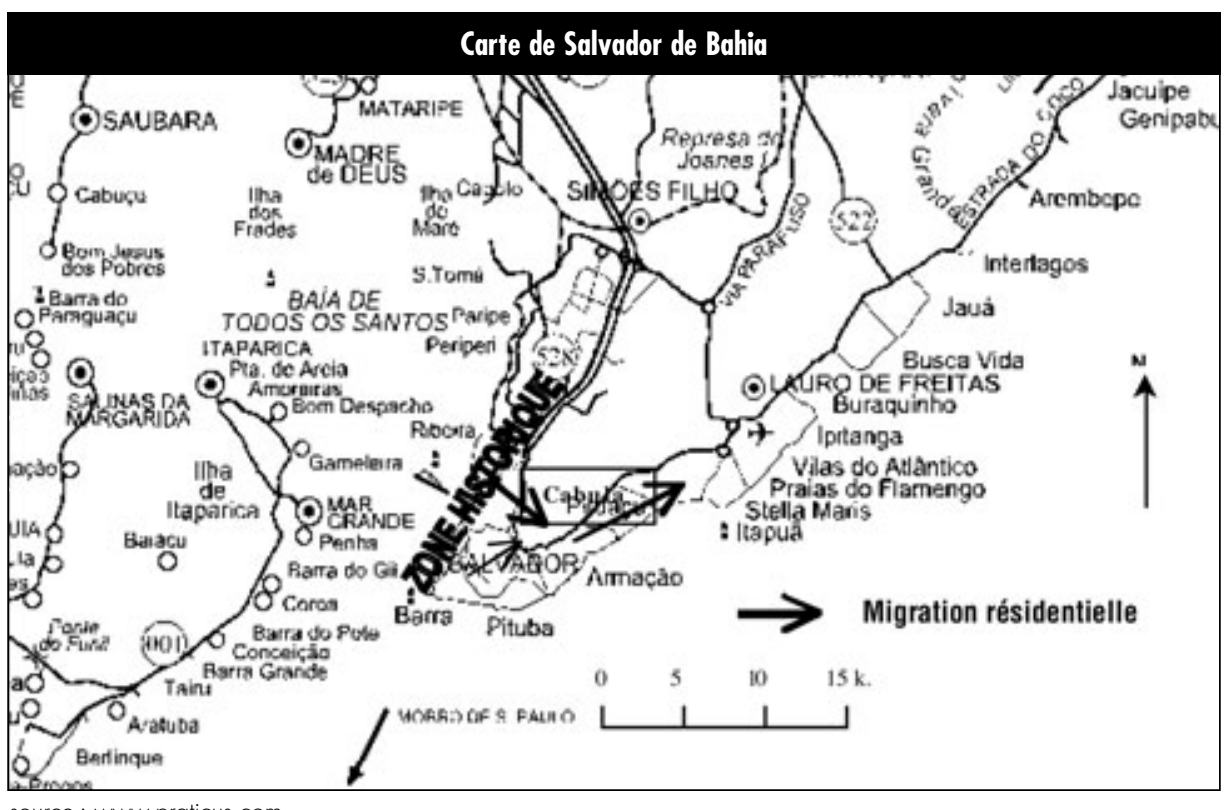

source: www.praticus.com 


\section{Panorama urbain}

À vue aérienne, le modernisme de la ville de Salvador s'affiche dans les édifices à logements et à bureaux qui s'élèvent (moins d'une vingtaine d'étages) au-dessus du centre de la péninsule, particulièrement la partie nord du quartier de Pituba. Le développement des tours gagne graduellement les quartiers les plus anciens. Les résidants des anciens quartiers (Brotas, Barra, Ondina) désespèrent tranquillement en constatant que le panorama océanique s'émiette de plus en plus. Les zones résidentielles se déplacent graduellement des quartiers anciens vers le centre nord et vers l'est, près des plages du quartier d'Itapoã (Agier, 1999: 303). Agier note par ailleurs que le déplacement résidentiel et cette occupation spatiale se produisent par regroupement selon une hiérarchie sociale engendrée par les métiers et les salaires fournis par les zones industrielles du nord de la ville. Il fait une apologie selon la couleur de la peau qui détermine un contexte d'organisation spatiale selon les revenus et les caractéristiques sociales : le noir aurait vraisemblablement moins accès au sommet hiérarchique professionnel par un plafonnement des postes et des salaires. Le manœuvre ou l'homme à tout faire de l'échelon inférieur ne peut se payer un logement dispendieux en bordure de mer; il choisit d'habiter le centre ou la périphérie nord de la ville.

Comment résoudre le problème en soulignant la couleur alors qu'il y a évidemment un métissage majoritaire? Certains avancent que des pourcentages de $40 \%$ des emplois, des études universitaires, des fonctions administratives les plus élevées, des propriétés privées ou autres avantages sociaux devraient être réservés aux noirs. Or, les noirs purs, selon les statistiques, ne représentent pas $40 \%$ de la population, mais plutôt $6 \%$. La discrimination raciale dure porterait donc sur ce pourcentage de la population, en s'amenuisant proportionnellement au blanchiment de la peau. À l'évidence, la complexité du métissage et la délicatesse du sujet exigent une approche plus nuancée que le simple décompte à partir de la couleur (simplification porteuse de racisme) $)^{7}$. Simplifier l'éventail racial en groupant les différentes couleurs en blancs et non-blancs cause un malaise, introduit par l'usage de la négation des nuances pour différencier des personnes beaucoup plus intéressantes que leur simple couleur. Mais la mise en rapport des conditions économiques et socioprofessionnelles pourrait révéler une problématique de regroupement et d'exclusion des communautés selon le revenu et l'origine ethnique.

À vue horizontale, en parcourant les rues, le clivage social se lit dans la répartition des quartiers résidentiels; la démarcation est claire et nette entre deux modes de vie qui coexistent difficilement. Chaque quartier bien organisé côtoie une favela (bidonville) s'agglutinant aux flancs des collines. L'espace péninsulaire, occupé par la ville, se constitue de talus, de collines et de plates-formes, laissant ainsi des parcelles de terrain vacantes, de moindre intérêt, envahies par des migrants pauvres. Ces espaces d'occupation spontanée dessinent des zones de minuscules logis (faits de briques rouge ocre tirées du sol occupé) entassés les uns sur les autres. Par l'occupation forcée et la résistance, une favela s'organise selon une structure et un fonctionnement autonome malgré le manque d'eau et de services municipaux, mais nécessairement parasitaire de l'oasis urbaine. La spontanéité de l'invasion occasionne, sur le plan urbain, des enchevêtrements imprévisibles qui correspondent mieux à la définition de sentiers pavés, de rues étroites sinueuses avec des dénivelées à la limite du glissement des terres (les pluies abondantes de la période d'hiver causent parfois des glissements appréhendés par les propriétaires vivant en contrebas).

Les murs de protection et les vigies à l'entrée des résidences des classes moyennes et riches tracent la limite de la zone à protéger contre les éventuels voleurs. Les soirées de Bahia, les plus tièdes et agréables au monde, sont singulièrement une invitation à entrer à la maison; la nuit exige une protection accrue, la rue se vide. L'accommodement capitaliste à l'usage de la pauvreté soustrait au capital beaucoup de vertus comme celle de profiter librement du charme et de l'exotisme de la ville. Selon Lannou (1976: 155), le paradoxe s'ex-

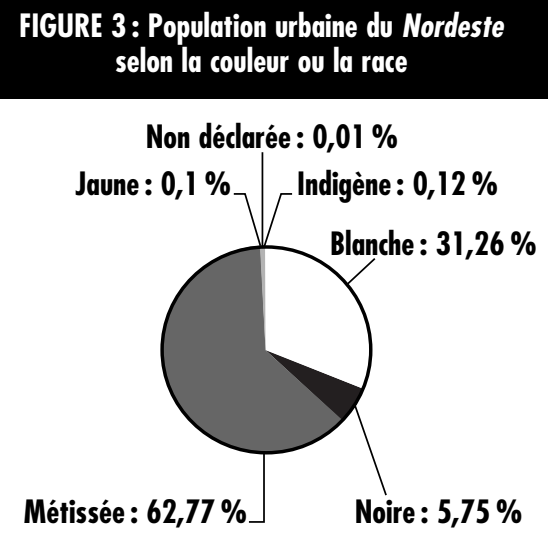

Source : IBGE

plique par l'assiette économique et sociale du Brésil, ce qui rejoint la problématique de Salvador :

Assiette est beaucoup dire : le Brésil n'en a guère d'assuré. Il a eu et il a encore des chances brillantes dans les compétitions des échanges internationaux, mais il lui manque toujours en dépit de ses enrichissements et de ses créations grandioses, la stabilité dans l'exploitation des ressources. Cette lacune tient à la persistance de certaines données psychologiques, au cloisonnement des groupes sociaux, mais aussi à l'insuffisante intégration des comportements nationaux par un système de transports singulièrement inachevé.

Il ajoute (p. 214) une observation qui souligne encore plus l'ambiguiité sociale :

La liberté dont témoignent les personnes et les conditions est loin d'exprimer leur liberté. Le travail des hommes au Brésil, porte encore les servitudes du passé colonial et le drame est que les activités les plus enrichissantes soient aussi celles qui maintiennent l'économie brésilienne dans une véritable sujétion.

Quant au réseau routier, à l'exception des ruelles secondaires, les principaux rues et boulevards sont acceptables, mais, à mesure que l'on s'éloigne du centre-ville, la plupart des routes régionales à la périphérie sont atroces et la signalisation quasi inexistante, le mélange de juridiction fédérale, 
étatique et municipale créant des vides de responsabilité. Simultanément, en traversant la ville vers la périphérie nord, on observe un effort des gouvernements à construire des ensembles administratifs et d'enseignement pour occuper l'intérieur des terres et faire en sorte de briser le paysage commercial, manufacturier et résidentiel chaotique. L'agglomération offre donc un développement économique, à la fois prospère et fragile, avec ses supermarchés de produits et services de luxe (Iguatemi, Itaigara, Aero Clube, Bom Preço) voisinant le mercantilisme artisanal du pauvre, qui s'approprie la rue, concurrençant sans complexe le commerce rutilant d'en face. Grâce à son ingéniosité, c'est heureux, le pauvre survit et se procure un peu de tout à un prix moindre. Deux types de marchés se superposent subtilement, le marché style américain et le marché populaire. Malgré tout, cette coexistence libérale des marchés locaux tolère la mendicité chez les enfants, les femmes et les adultes. Même si l'on trouve cette mendicité dans la plupart des grandes agglomérations mondiales et brésiliennes, à Salvador ce n'est pas une mendicité ponctuelle d'agrément, mais souvent existentielle.

Quels sont les déterminants qui ont abouti à élaborer une telle structure urbaine et contrastée du tissu social? Répondre c'est répéter le qualificatif de Lannou (1976: 95), à savoir que Salvador, à la suite de son accroissement démographique (40 000 habitants/an) depuis 1940, s'est agrandie comme «une véritable enflure urbaine» sans commune mesure avec les ressources locales disponibles. Souscrire à cette affirmation c'est considérer Salvador comme un cas unique, alors que São Paulo, Rio de Janeiro et les autres villes brésiliennes connaissent en soi les mêmes difficultés, sinon avec une acuité plus extrême encore. Selon les statistiques, la majorité des agglomérations urbaines brésiliennes les plus importantes déclarent la présence de favelas, d'habitations non conformes, d'illégaux, (IBGE, 2001 : 95). Dans une vision élargie, ce phénomène des bidonvilles, aussi généralisé, s'amplifie avec le processus mondial d'urbanisation (migration rurale/industrialisation). Ce phénomène crée nécessairement un bassin de main-d'œuvre bon marché. Le laxisme des autorités politiques collabore en soi à l'implantation d'un tel type d'habitat communautaire qui, avec le temps, se légalise, se structure, se personnalise. Chaque favela est donc une entité urbaine en devenir, un chantier social spécifique selon les lieux, les origines et l'histoire (Pebayle, 1995 : 62-66).

\section{Rayonnement économique de Salvador}

La position géographique de la ville fut avantageuse dans le passé, grâce à sa centralité comme point commercial et de distribution agricole. Hypothétiquement, cette prédisposition dans l'axe de circulation pourrait l'avantager dans l'avenir, grâce à la logique d'expansion territoriale d'un centre métropolitain régional de commerce et de distribution (Droulers, 2001: 231). Avec le support politique et un cycle économique favorable au Nordeste, l'agglomération peut redevenir un noyau de transbordement et d'échange intéressant pour les marchés intérieurs et extérieurs.

Salvador a profité de son avantage urbain pour séduire la grande majorité (Théry, 1995 : 149; Lannou, 1976 : 94 ; Agier, 1999: 299-304) des travailleurs des complexes pétrochimiques et industriels de Camaçari et d'Aratu, quoique situés à quarante kilomètres au nord. Elle bénéficierait des caractéristiques attractives d'un pôle régional. C'est un indice qu'avec une meilleure infrastructure des voies de communication et une rénovation urbaine accrue (telle qu'a connue récemment le quartier de Pituba par exemple), doublées d'une protection de la qualité des plages, toute activité satellite serait en partie polarisée vers la troisième métropole brésilienne (la fixation des limites des villes brésiliennes varie selon les paliers d'administration).

\section{Conclusion}

Malgré la montée industrielle rapide des années 1950 du triangle Belo Horizonte, São Paulo, Rio de Janeiro, l'activité économique de Salvador n'est plus nécessairement soumise simplement au cycle de production du sucre, du coton ou de la viande et au commerce international. Un éventuel regain (hydroélectricité, voies de communications, irrigation) dans la partie nord, celle du Sertão (région de grandes promesses) environnant les rives du Rio São Francisco pourrait lui redonner cette vitalité naguère perdue comme centre de redistribution agricole. Toute croissance ou mouvement économique de l'ouest et de l'intérieur du Brésil amazonien ne peut que se propager proportionnellement à la zone d'influence de la métropole bahianaise, en considérant sa situation géographique centrale face au São Francisco, grâce à son poids démographique accru, grâce à son infrastructure portuaire et de services.

Le temps joue en sa faveur et l'industrie lourde n'est peut-être pas le moteur éventuel du renouvellement, mais un gain significatif dans le secteur industriel de pointe et le secteur tertiaire (commercial, administratif, social, environnement) à la fois protégerait sa spécificité et assurerait son développement durable. Elle a été épargnée par les cheminées fumantes; à chaque jour son ciel est aussi clair, libre de pollution et de retombées toxiques.

Progressivement, la hausse du taux de fréquentation scolaire et de scolarisation, particulièrement dans les classes sociales les moins favorisées ${ }^{8}$, l'augmentation du nombre de petites et moyennes entreprises (productrices d'un grand nombre d'emplois), une capitalisation dans l'entrepreneurship (ingéniosité mercantile) sont tous des facteurs susceptibles de produire une meilleure répartition des revenus. Le cloisonnement social ne peut résister à la tolérance brésilienne. La pire hypothèse à envisager est la formation permanente de ghettos de classes économiques qui occasionnent perpétuellement des oppositions coûteuses. Les traditions d'unité nationale du système d'organisation brésilien, malgré le régionalisme économique, incorporent des automatismes nationaux démocratiques fonctionnels récemment acquis. L'élection récente du PT (Parti des Travailleurs) est une indication et une orientation qui encourage la majorité à appliquer progressivement des principes d'intégration des groupes marginaux par une meilleure accessibilité aux terres et aux revenus. 
En matière de problèmes internes de gestion, on peut noter des obstacles à la mise en valeur de l'actif de Salvador de Bahia quant à l'offre internationale de ses ressources. L'usage quasi exclusif de la langue portugaise pour accueillir les étrangers est un frein de taille au marché extérieur. Une intensification de l'apprentissage des principales langues étrangères n'exige pas de mises de fond extraordinaires, la préfecture peut encourager et favoriser les institutions à établir des départements de langues orientés sur les affaires et les relations internationales. L'autarcie tranquille, se fiant particulièrement à l'activité économique interne, assure une stabilité, mais une revitalisation des échanges internationaux pourrait relancer une période de croissance similaire à celle de la période 1960-1990.

La protection de l'environnement, une sécurité civile améliorée, une communication plus adaptée au reste du monde supportent une relance du secteur tertiaire consolidé par des services sociaux et éducatifs appropriés. La mise en marché de la ville touristique de Salvador semble un défi de tous les saints, gratifiant et merveilleux.

Le cachet touristique de la ville, fondé sur la régularité de son climat et sur une riche historicité architecturale, réside aussi dans le folklore et la personnalité du citoyen. Citoyen qui partage volontiers son temps entre son travail et les activités de plage : la cité profondément brésilienne lui ressemble dans sa manière artistique de s'exprimer (la mode vestimentaire, le chant, la danse, etc.). Cet abandon tranquille au temps nous impose un sentiment (vérifié ou non) que la poésie bahianaise mène à l'harmonie et à la connaissance ; demain la cité va s'achever, aujourd'hui, c'est la fête avec l'espérance des dieux. Le mythe urbain solteropolitain (épithète des gens qui habitent la ville de Salvador) se concrétise, la ville domine la mer, mais aussi son histoire, par sa position privilégiée, ses gains en alphabétisation, sa protection et la revalorisation observable de son héritage culturel architectural.
L'émancipation économique de Salvador représenterait une réussite non seulement pour le Brésil, mais un enrichissement de la communauté internationale. Sinon, la mise en marché du mysticisme recèle des servitudes du pauvre qui accepte de rejouer la boucle infinie du fatalisme social.

Yvan Desbiens, est professeur et chercheur associé au Département des sciences humaines, Université du Québec à Chicoutimi $(U Q A C)$.

\section{Notes}

1 Le noir pur représenterait près de $6 \%$ de la population, le métissage a produit un blanchiment selon les données de l'IBGE (voir figure 3).

2 Par exemple (Théry, 1995 : 126), dans le sertão du São Francisco en 1711, l'étendue de la propriété monarchique de la Casa da Torre atteignait 1500 kilomètres.

3 Dans un article du journal $O$ Globo (03/12/03), le ministre de l'Éducation révèle que 159580 jeunes bahianais de sept à quatorze ans ne fréquentent pas encore l'école.

4 Religion qui emprunte des rituels africains et associe les éléments naturels (terre, eau, forêt...) à des divinités respectives.

5 L'Axé music est une salutation religieuse associée au camdomblé et au circuit musical soteropolitano (de Salvador).

6 Sur la page du site Internet de la préfecture, on peut lire que «la cité maintient son caractère prestataire de services dans les activités bancaires, commerciales et l'exportation de produits régionaux : le sucre, le cacao et la viande fumée. De plus, selon les données de CONDER [compagnie de développement urbain de l'État de Bahia] en 1997, approximativement $85 \%$ des emplois de la région métropolitaine de Salvador seraient dans le secteur tertiaire et ce pourcentage irait en s'accroissant. »

7 Walniez et Brustlein (2000: 113) observent la difficulté de classer la population de noire ou de blanche au Brésil, en raison du métissage important.

8 Globo news.com le 12 décembre 2003: selon les données de l'IBGE 2003, les blancs ont cinq fois plus de chance d'accéder aux études supérieures.

\section{Bibliographie}

Agier, Michel (1999), «Une Ville entre magie et industrie: Nouveaux espaces d'identité à Bahia », Problèmes d'Amérique latine, $\mathrm{n}^{\circ} 14$, juillet-septembre.

Dos Passos, John (1964), Le Brésil en marche, Collection L'air du temps, Gallimard.

IBGE (Instituto Brasileiro de Geografia e Estatística) (2001), Perfil dos Municipios Brasileiros, p. 95.

Droulers, Martine (2001), Brésil: une géohistoire, Presses universitaires de France.

Lannou, Maurice (1955), Le Brésil, Armand Colin.

Lannou, Maurice (1976), Le Nouveau Brésil, Armand Colin.

Martins Chalub, Leila (1998), Aspectos sociais e Antropológicos Desenvolvimento Sustentàvel : O Casodo Acampamento do Trabalhadores Rurais Sem terra de Água Fria-Goiás, Tendência da Educação ambiental Brasileira, UNISC, p. 145-163.

Mauro, Frédéric (1975), L’Amérique espagnole et portugaise de 1920 à nos jours, Presses universitaires de France.

Pebayle, Raymond (1995), Le Brésil, que sais-je?, Presses universitaires de France.

Santos, Milton (1968), «Croissance urbaine et nouvelle armature urbaine au Brésil », Annales de géographie, janvier-février.

Site de la préfecture de Salvador, [http://www.pms.ba.gov.br/indexN800.html].

Smith, T.L., Brazil, People and Institution, Louisiana State University Press, 1972.

Soares de Souza, Licia (1999), Des récits régionalistes à la télévision: La voix de la Terre au Québec et au Brésil, Canadart VII, ABECAN, p. 120-127.

Théry Hervé (1995), Pouvoir et territoire au Brésil, De l'archipel au continent, Éditions de la maison des sciences de l'homme, Paris,

Walniez, Philippe, et Violette Brustlein (2000), «La différenciation sociale et spatiale des religions au Brésil », Cahiers des Amériques Latines, vol. 33, p. 111-113. 